\title{
A Robot-based Gait Training System for Post-Stroke Rehabilitation
}

\author{
Sharon Banh ${ }^{1}$, Emily Zheng ${ }^{2}$, Alyssa Kubota ${ }^{1}$, Laurel D. Riek ${ }^{1}$ \\ ${ }^{1}$ Computer Science and Engineering, University of California San Diego \\ ${ }^{2}$ Computing and Mathematical Sciences, California Institute of Technology
}

\begin{abstract}
As the prevalence of stroke survivors increases, the demand for rehabilitative services will rise. While there has been considerable development in robotics to address this need, few systems consider individual differences in ability, interests, and learning. Robots need to provide personalized interactions and feedback to increase engagement, enhance human motor learning, and ultimately, improve treatment outcomes. In this paper, we present 1) our design process of an embodied, interactive robotic system for post-stroke rehabilitation, 2) design considerations for stroke rehabilitation technology and 3) a prototype to explore how feedback mechanisms and modalities affect human motor learning. The objective of our work is to improve motor rehabilitation outcomes and supplement healthcare providers by reducing the physical and cognitive demands of administering rehabilitation. We hope our work inspires development of human-centered robots to enhance recovery and improve quality of life for stroke survivors.
\end{abstract}

\section{ACM Reference Format:}

Sharon Banh, Emily Zheng, Alyssa Kubota, Laurel D. Riek. 2021. A Robotbased Gait Training System for Post-Stroke Rehabilitation. In Companion of the $2021 \mathrm{ACM}$ /IEEE International Conference on Human-Robot Interaction (HRI'21 Companion), March 8-11, 2021, Boulder, CO, USA. ACM, NY, NY, USA, 5 pages. https://doi.org/10.1145/3434074.3447212

\section{INTRODUCTION}

Stroke is a leading cause of death and disability [7]. It is estimated that by 2030, there will be 77 million stroke survivors worldwide [29]. About $80 \%$ of stroke survivors experience motor impairment which severely affects their ability to live independently and perform activities of daily living such as eating and bathing [18, 22].

Rehabilitation greatly impacts recovery post-stroke, as stroke survivors can regain physical function and independence [21]. Rehabilitation aims to help survivors relearn the skills lost due to stroke, but it can vary widely depending on an individual's affected skills and level of severity. For example, speech therapy aim to improve a person's language, communication, or swallowing abilities, whereas physiotherapy interventions aim to improve their balance, gait, and movement [17].

Research reported in this paper is supported by the National Science Foundation under CMMI-1935500 and the Computing Research Association DREU program.

Permission to make digital or hard copies of part or all of this work for personal or classroom use is granted without fee provided that copies are not made or distributed for profit or commercial advantage and that copies bear this notice and the full citation on the first page. Copyrights for third-party components of this work must be honored For all other uses, contact the owner/author(s).

HRI '21 Companion, March 8-11, 2021, Boulder, CO, USA

(c) 2021 Copyright held by the owner/author(s).

ACM ISBN 978-1-4503-8290-8/21/03.

https://doi.org/10.1145/3434074.3447212
However, rehabilitation may take years of consistent monitoring and training to show discernible results [17]. Also, like any intervention, personalization is critical to ensure adherence [20,35].

Researchers have developed many robotic devices to improve post-stroke rehabilitation. Robots are well-suited to the repetitive, task-oriented nature of many rehabilitation treatments, and they may alleviate a therapist's physical and cognitive load as compared to traditional treatment approaches [31]. In addition, robots offer a controlled means of administering treatment, and can precisely measure an individual's performance and progress [15, 31]. However, there is inconsistent evidence regarding the efficacy of robot-assisted rehabilitation as compared to traditional methods [24, 31].

Furthermore, few systems account for individual differences or support personalized, adaptive interactions, despite their importance $[25,30,36]$. Previous research has demonstrated that task difficulty, feedback modality, and feedback designs can be manipulated to an individual's specific level of skill to optimize motor learning [12]. However, it is unclear which feedback strategies, and modality or combination of modalities can best enhance motor learning and support human autonomy, particularly in the context of robot-assisted rehabilitation [19]. This represents a gap and unmet need obstructing the development of intelligent robotic systems for motor rehabilitation.

To address this gap, we are developing an embodied, intelligent robotic system to provide adaptive performance feedback for stroke survivors receiving gait rehabilitation. We aim to discern the relationship between various performance feedback approaches and long-term motor retention in the context of gait rehabilitation.

The contributions of this work are threefold. First, we present the design for a novel interactive robotic feedback system, developed through a co-design process with human motor learning experts. Second, we introduce design considerations for rehabilitation robots to assist older adults engaging in post-stroke rehabilitation. Finally, we propose a prototype of an embodied, interactive robot that provides non-contact assistance during post-stroke rehabilitation. Ultimately, using our system, we hope to determine how robotbased feedback strategies affect and are perceived by individuals to ultimately improve the efficacy of rehabilitation.

\section{BACKGROUND}

\subsection{Feedback Personalization}

Personalizing robot feedback and behavior to an individual's performance, preferences, and goals is critical to improving the efficacy of robot-assisted motor rehabilitation [24]. Providing feedback based on a user's performance can significantly impact learning, retention, and engagement during motor rehabilitation among stroke survivors [20, 26, 35]. Moreover, the type of feedback (e.g. reward- 

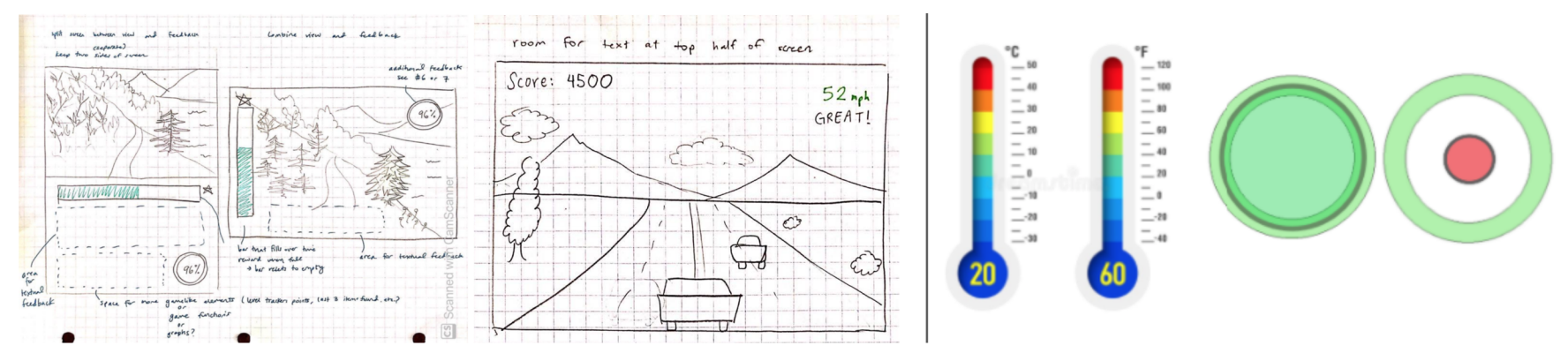

Figure 1: Left: Storyboards of game designs. Right: Non-gaming feedback mechanisms.

or punishment-based) can impact a user's rate of learning and retention in motor learning. For example, punishment-based feedback has been shown to accelerate immediate learning, while rewardbased feedback can increase retention of motor memory [9].

Reward- and punishment-based feedback has also been used in game settings. In fact, there has been considerable research done on the use of games in rehabilitation that show that game-based rehabilitation can improve acceptance and engagement [23].

Despite recent advancements, there are still open questions about how robots can best provide effective feedback to enhance motor recovery. Furthermore, current rehabilitation robots lack the capability of delivering personalized treatment and interactions. Thus, the goal of our work is to develop an embodied, intelligent robotic system that provides personalized performance feedback during post-stroke gait rehabilitation and compare how different feedback approaches impact human motor learning.

\subsection{Robotics for Motor Rehabilitation}

Rehabilitation robots range from providing physical assistance to social assistance and supporting upper and lower limb rehabilitation [27]. In motor rehabilitation where a robot physically assists a person, the person typically repeats some motion with the aid of robotic actuators rather than a human therapist [24]. These systems may reduce the physical strain and workload of therapists by providing high-intensity, task-specific training.

Because motor rehabilitation can become boring or frustrating, particularly over long periods of time [4], it is crucial to provide stroke survivors with social support in addition to physical support. Therefore, researchers are increasingly exploring the use of robots such as Pepper and NAO to provide non-contact social support to enhance rehabilitation $[4,8,34]$. Physically embodied robots can help motivate users, improving engagement with treatment and thus the treatment's efficacy $[5,6,8,34]$.

Many of these robots can automatically provide feedback based on a person's performance during treatment. For instance, researchers programmed a NAO robot to give verbal feedback and encouragement based on performance (e.g. posture) and physiological measurements (e.g. heart rate), as well as alert the therapist of unusual behavior [4]. However, to our knowledge, the relationship between feedback type and motor learning in the context of post-stroke gait rehabilitation is still unknown. Thus in our work, we focus on how robotic-based performance feedback impacts motor learning and retention among stroke survivors receiving gait rehabilitation.

\section{DESIGN APPROACH}

Our objective is to design an interactive robotic system to explore how various feedback mechanisms and modalities affect motor learning during post-stroke gait rehabilitation. We first reviewed prior work on game design for stroke rehabilitation, collaborated with neurorehabilitation researchers and practitioners studying gait, and engaged in participatory design with older adults. Through a review of the stroke game design literature, we identified several considerations that would inform the design of our application.

\subsection{Design Considerations For Designing Stroke Rehabilitation Technology}

There has been considerable research done on the use of games in rehabilitation that show game-based rehabilitation can improve acceptance and engagement [23]. Because $75 \%$ of all strokes occur in adults older than 65 years old, our designs needed to consider the preferences of this age group.

Visual Simplicity: As age increases, static and dynamic acuity diminishes, making it difficult for people to perceive small elements and locate information. Thus, images and text must be appropriately sized and clearly visible, and interfaces should not be cluttered so as to not confuse older adults unaccustomed to digital games [13].

Contrasting Colors: While colorful scenes can increase user engagement and attention, there must be appropriate color contrast to consider the loss of color sensitivity associated with aging [2, 28].

Personalization: Training with personalized difficulty levels leads to higher levels of engagement and enhanced learning outcomes, compared to fixed difficulty $[10,16,33]$. Engagement is essential to treatment adherence and better treatment outcomes.

\subsection{Ideation}

3.2.1 Development of Stroke Gait Rehabilitation Game Ideas. Gamebased learning involves using game play to achieve a specific learning outcome. We explored various game designs that may be appropriate for gait rehabilitation and could be played on our robot's tablet (see Section 3.3). Some of our design concepts included a race car game, an alien invasion defense game, and a virtual walk (see Figure 1, Left). For each concept, we devised game objectives, level progression, and quantitative incentive structures.

3.2.2 Development of Feedback Mechanisms in a Non-Gaming Context. Gamification is the use of game elements such as leaderboards, progress bars, and point-based incentives in non-game contexts to 


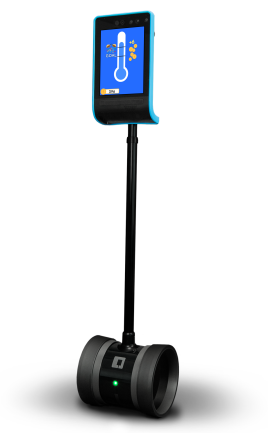

Figure 2: Our interactive robotic system for rehabilitation.

engage and motivate users to complete a task. In comparison to playing an actual game, gamification can be less cognitively tasking. Thus, we also developed feedback mechanisms using gamification tools. For example, a thermometer bar or target circle with an ideal range can denote when a participant has met a goal for each step taken (see Figure 1, Right). Additionally, overall progress in the session can be represented by earning stars or circles.

\subsection{System Design Proof of Concept}

We designed an adaptive robotic system aimed at providing realtime performance feedback through non-contact interaction (nonverbal visual and gestural communication). Our system is embodied on a Double, which is a tablet-based telepresence robot (see Figure 2). These robots are readily available and in use in many settings, including schools, offices, and hospitals, which may improve acceptability and trust in rehabilitation settings. Furthermore, prior studies have shown that tablets have high acceptability, encourage participation, and contribute to engagement in stroke rehabilitation $[11,32]$. Thus, as a tablet-based robot, the Double has the potential advantages of being engaging, interactive, and easy to use.

The robot will be integrated into a gait rehabilitation program facilitated by researchers who focus on motor recovery following stroke. Using reward, punishment, or neutral behaviors, the robot will supplement human-delivered therapy by providing noncontact performance feedback based on the motor performance of the participant. Motor performance will be measured by the ankle force produced by a walking participant. Each session will begin with a baseline assessment to evaluate motor performance during a walking task. After establishing a participant's baseline, feedback should indicate when the participant has met or exceeded the baseline, as well as a predetermined performance goal.

\subsection{Design Workshop 1}

To assess potential designs and establish design requirements, we held a workshop with motor learning researchers and rehabilitation specialists. We determined three design requirements.

Conceptual Simplicity: The interface design and feedback mechanism should be simple and easily understandable, particularly to older adults who may have low technology literacy.

Continuous Feedback: Feedback should be provided continuously in real-time throughout a session so participants learn and adjust their behavior.

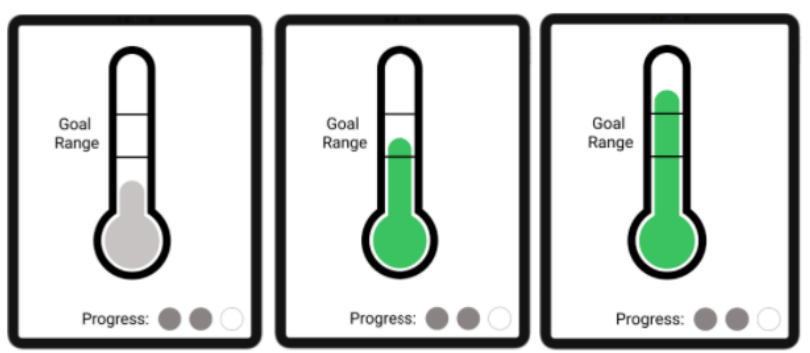

Figure 3: One design iteration of the thermometer feedback system. We aimed to maintain a simple aesthetic, with a white background and minimal text.

Concrete Goals: Participants should have a concrete goal, and feedback should clearly indicate performance relative to that goal.

To satisfy these design requirements, we considered gamification mechanics such as those discussed in Section 3.2.2. Together with the motor learning researchers and rehabilitation specialists, we determined that a thermometer feedback mechanism would best satisfy these requirements. A thermometer can visually display a participant's motor performance relative to their baseline and goal performance thresholds. Thus, for each step, a participant can easily see if they reached their goal, or how far they were from their goal.

\subsection{Design Iterations}

We developed a straightforward feedback system design where a thermometer bar models a participant's ankle force, and filled and unfilled circles measure overall progress in the session. Figure 3 demonstrates the reward condition. Here, the height of the thermometer represents the real-time measurement of force produced by a participant's ankle. When a participant meets the baseline value, the thermometer changes from grey to green. On the thermometer bar, the participant will see that the lower black line represents the baseline value that will be measured at the start of each session. The goal of the participant is to reach the top line, a $15 \%$ increase of ankle force based on the measured baseline value.

Circles at the base of the screen represent a participant's progress in their overall session. Progress is assessed by the percentage of total step attempts that met the specified goal (e.g. if 8 steps met the ankle force goal out of the total 10 step attempts, the player has an $80 \%$ accuracy in meeting the specified goal). If there are 3 possible circles to be earned: earning one circle could represent 0-40\% accuracy, two circles could represent $40-70 \%$, three circles could represent $70-100 \%$ accuracy.

\subsection{Design Workshop 2}

Given that the incidence of stroke increases with age and mainly occurs in older adults, we led semi-structured interviews with four individuals $(n=4)$ older than 50 years old to gather feedback on our static interface. Half of these participants were female, and none had experienced a stroke. Participants provided feedback regarding how our designs could better appeal to our target audience and satisfy the design considerations established in Sections 3.1 and 3.4. 

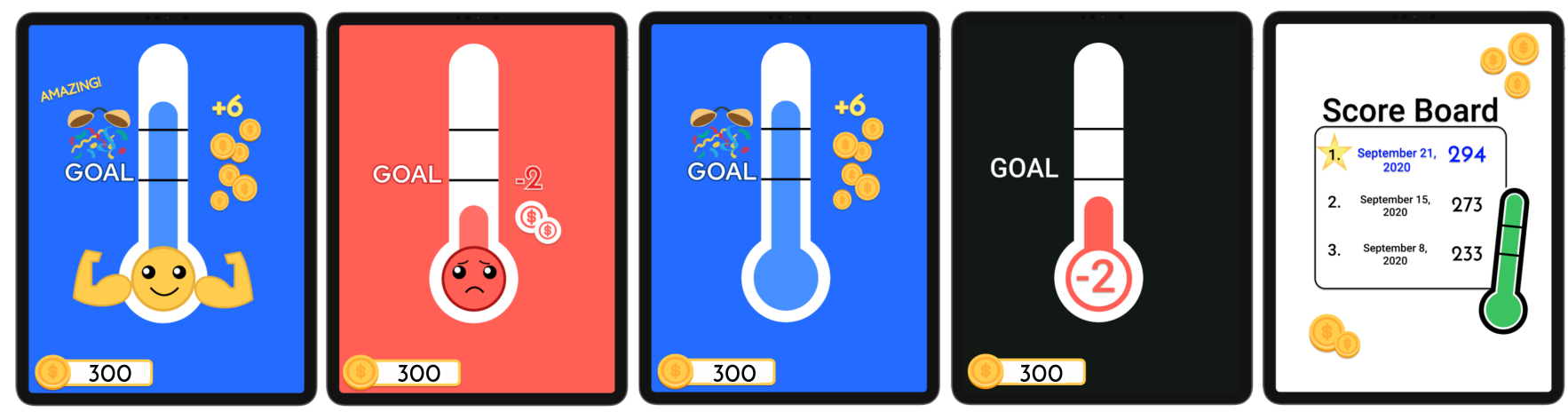

Figure 4: Various designs of the thermometer feedback system. We filled the background to improve visibility of color and implemented a point-based system to represent progress.

\subsection{Current Designs}

Our current designs implement feedback from motor learning researchers, rehabilitation specialists, and individuals older than 50 years old while reflecting the design considerations stated in Sections 3.1 and 3.4 (see Figure 4).

Visual Simplicity: While designing the interfaces for our tablet, we considered the possibility that the interface could over-stimulate older adults and be a distraction from the treatment. Therefore, we designed multiple variations of our interface with varying levels of visual stimuli. To design a visually accessible feedback system, we considered the Web Content Accessibility Guideline (WCAG) 2.0 Level AA guidelines for color contrast [3].

Contrasting Colors: There was a consensus that the design should incorporate color and be more visually engaging. To add more color to the design and increase the visibility of color, we filled the background to match the internal color of the thermometer. We also changed the red color to be a lighter hue because we received feedback that it was harsh on the eyes. Furthermore, we used blue to represent a positive achievement, rather than green because blue has been shown to increase feelings of calmness and relaxation [1]

Personalization: We created multiple designs that may appeal to individual preferences. For instance, we designed variations with smiling and frowning faces to enhance the social presence of our robot, and possibly contribute to its acceptability and likability [37].

In addition, it is possible that stroke survivors may also prefer a dark-mode variation of the thermometer feedback system. A recent study of different vision modes for AR found that the light-on-dark scheme was preferred, and significantly increased visual acuity and reduced visual fatigue (e.g. light images on a dark background) [14].

Conceptual Simplicity: As discussed in Section 3.2.2, gamification is the use of game elements such as point-based incentives and leaderboards to promote task engagement. Based on feedback on our prior design, our stakeholders found the progress circles confusing. So, we developed a score system where participants could earn a specified number of points based on the condition of the experiment and the performance of each step taken. We also designed a scoreboard to show participants their progression between sessions and motivate them to improve their performance.
Continuous Feedback: The score system also addresses the importance of frequently providing feedback to participants. Participants receive immediate performance feedback for each step, as indicated by the deduction or addition of points.

Concrete Goals: Participants also expressed the need for differentiation between meeting the target value and exceeding the target value. For that reason, we incorporated a celebratory icon and encouraging text in one of our variations.

\section{PLANNED WORK}

Robot Feedback. As we continue to develop our system, we plan to design and implement different robotic feedback gestures. These robot gestures will focus on physical movement, such as moving back and forth or adjusting its height. These movements will vary for each condition in correspondence with the nonverbal visual feedback provided by the tablet.

Tablet-based Feedback. We will gather feedback through co-design sessions with stroke survivors, therapists, and neurorehabilitation researchers to inform future iterations of the system. Once we have finalized our visual designs with stakeholders, we plan to implement the designs on a tablet.

Longitudinal Studies. After the system is complete, we will perform longitudinal studies with stroke survivors undergoing gait rehabilitation to investigate how different forms and combinations of performance feedback affect motor learning.

\section{DISCUSSION}

It is crucial to understand the underlying mechanisms of motor learning from various forms of feedback. Our objective is to develop an adaptive robot that provides personalized feedback to improve rehabilitation outcomes for stroke survivors.

In this work, we presented our first prototype for an interactive robotic system that provides real-time visual and behavioral performance feedback to stroke survivors during post-stroke gait rehabilitation. We employed a user-centered and participatory approach to design an effective and safe rehabilitation robot. Our work aims to advance the efficacy of robot-based rehabilitation in general, and we hope it encourages researchers to examine the role of adaptive personalization for rehabilitation robotics. 


\section{REFERENCES}

[1] A. AL-Ayash, R. T. Kane, D. Smith, and P. Green-Armytage. The influence of color on student emotion, heart rate, and performance in learning environments. Color Research \& Application, 41(2):196-205, 2016.

[2] G. Alankus, A. Lazar, M. May, and C. Kelleher. Towards customizable games for stroke rehabilitation. In Proceedings of the SIGCHI conference on human factors in computing systems, pages 2113-2122, 2010.

[3] B. Caldwell, M. Cooper, L. G. Reid, G. Vanderheiden, W. Chisholm, J. Slatin, and J. White. Web content accessibility guidelines (wcag) 2.0. WWW Consortium (W3C), 2008.

[4] N. Céspedes, M. Múnera, C. Gómez, and C. A. Cifuentes. Social human-robot interaction for gait rehabilitation. IEEE Transactions on Neural Systems and Rehabilitation Engineering, 2020.

[5] E. Deng, B. Mutlu, and M. Mataric. Embodiment in socially interactive robots. arXiv preprint arXiv:1912.00312, 2019.

[6] J. Fasola and M. J. Matarić. A socially assistive robot exercise coach for the elderly Journal of Human-Robot Interaction, 2(2):3-32, 2013.

[7] V. L. Feigin, B. Norrving, and G. A. Mensah. Global burden of stroke. Circulation research, 120(3):439-448, 2017

[8] R. Feingold Polak and S. L. Tzedek. Social robot for rehabilitation: Expert clinicians and post-stroke patients' evaluation following a long-term intervention. In Proceedings of the 2020 ACM/IEEE International Conference on Human-Robot Interaction, pages 151-160, 2020.

[9] J. M. Galea, E. Mallia, J. Rothwell, and J. Diedrichsen. The dissociable effects of punishment and reward on motor learning. Nature neuroscience, 18(4):597-602, 2015.

[10] K. M. Gerling, R. L. Mandryk, and C. Linehan. Long-term use of motion-based video games in care home settings. In Proceedings of the 33rd Annual ACM Conference on Human Factors in Computing Systems, pages 1573-1582, 2015.

[11] S. R. Greysen, R. R. Khanna, R. Jacolbia, H. M. Lee, and A. D. Auerbach. Tablet computers for hospitalized patients: a pilot study to improve inpatient engagement. Journal of hospital medicine, 9(6):396-399, 2014.

[12] M. A. Guadagnoli and T. D. Lee. Challenge point: a framework for conceptualizing the effects of various practice conditions in motor learning. Fournal of motor behavior, 36(2):212-224, 2004.

[13] W. Ijsselsteijn, H. H. Nap, Y. de Kort, and K. Poels. Digital game design for elderly users. In Proceedings of the 2007 conference on Future Play, pages 17-22, 2007.

[14] K. Kim, A. Erickson, A. Lambert, G. Bruder, and G. Welch. Effects of dark mode on visual fatigue and acuity in optical see-through head-mounted displays. In Symposium on Spatial User Interaction, pages 1-9, 2019.

[15] A. Kubota, E. I. Peterson, V. Rajendren, H. Kress-Gazit, and L. D. Riek. Jessie: Synthesizing social robot behaviors for personalized neurorehabilitation and beyond. In Proceedings of the 2020 ACM/IEEE International Conference on HumanRobot Interaction, pages 121-130, 2020.

[16] A. Kubota and L. D. Riek. Methods for robot behavior adaptation for cognitive neurorehabilitation. Annual Review of Control, Robotics, and Autonomous Systems, 2021.

[17] P. Langhorne, J. Bernhardt, and G. Kwakkel. Stroke rehabilitation. The Lancet, 377(9778):1693-1702, 2011

[18] P. Langhorne, F. Coupar, and A. Pollock. Motor recovery after stroke: a systematic review. The Lancet Neurology, 8(8):741-754, 2009.

[19] H. R. Lee and L. D. Riek. Reframing assistive robots to promote successful aging. ACM Transactions on Human-Robot Interaction (THRI), 7(1):1-23, 2018.
[20] M. H. Lee, D. P. Siewiorek, A. Smailagic, A. Bernardino, and S. B. Badia. Towards personalized interaction and corrective feedback of a socially assistive robot for post-stroke rehabilitation therapy. In 2020 29th IEEE International Conference on Robot and Human Interactive Communication (RO-MAN), pages 1366-1373. IEEE, 2020.

[21] C. Mahak, Y. Shashi, N. M. Hemlata, G. Sandhya, K. Dheeraj, M. Dhandapani, and S. Dhandapani. Assessment of utilization of rehabilitation services among stroke survivors. Journal of neurosciences in rural practice, 9(4):461, 2018.

[22] L. Mercier, T. Audet, R. Hébert, A. Rochette, and M.-F. Dubois. Impact of motor, cognitive, and perceptual disorders on ability to perform activities of daily living after stroke. Stroke, 32(11):2602-2608, 2001.

[23] O. Mubin, F. Alnajjar, A. Al Mahmud, N. Jishtu, and B. Alsinglawi. Exploring serious games for stroke rehabilitation: a scoping review. Disability and Rehabilitation: Assistive Technology, pages 1-7, 2020.

[24] A. Pennycott, D. Wyss, H. Vallery, V. Klamroth-Marganska, and R. Riener. Towards more effective robotic gait training for stroke rehabilitation: a review. fournal of neuroengineering and rehabilitation, 9(1):1-13, 2012.

[25] Z. Qian and Z. Bi. Recent development of rehabilitation robots. Advances in Mechanical Engineering, 7(2):563062, 2015.

[26] G. Quattrocchi, R. Greenwood, J. C. Rothwell, J. M. Galea, and S. Bestmann. Reward and punishment enhance motor adaptation in stroke. fournal of Neurology, Neurosurgery \& Psychiatry, 88(9):730-736, 2017.

[27] L. D. Riek. Healthcare robotics. Communications of the ACM, 60(11):68-78, 2017.

8. Shapi'i, N. N. Bahari, H. Arshad, N. A. M. Zin, and Z. R. Mahayuddin. Rehabilitation exercise game model for post-stroke using microsoft kinect camera. In 2015 2nd International Conference on Biomedical Engineering (ICoBE), pages 1-6. IEEE, 2015.

[29] K. Strong, C. Mathers, and R. Bonita. Preventing stroke: saving lives around the world. The Lancet Neurology, 6(2):182-187, 2007.

[30] Z. Wang, M. K. Singh, C. Zhang, L. D. Riek, and K. Chaudhuri. Stochastic multiplayer bandit learning from player-dependent feedback. In ICML Workshop on Real World Experiment Design and Active Learning, 2020.

[31] L. M. Weber and J. Stein. The use of robots in stroke rehabilitation: A narrative review. NeuroRehabilitation, 43(1):99-110, 2018.

[32] J. White, H. Janssen, L. Jordan, and M. Pollack. Tablet technology during stroke recovery: a survivor's perspective. Disability and rehabilitation, 37(13):1186-1192, 2015.

[33] C. D. Wickens, S. Hutchins, T. Carolan, and J. Cumming. Effectiveness of part-task training and increasing-difficulty training strategies: a meta-analysis approach. Human Factors, 55(2):461-470, 2013.

[34] R. Wilk and M. J. Johnson. Usability feedback of patients and therapists on a conceptual mobile service robot for inpatient and home-based stroke rehabilitation. In 5th IEEE RAS/EMBS international conference on biomedical robotics and biomechatronics, pages 438-443. IEEE, 2014

[35] K. Winkle, P. Caleb-Solly, A. Turton, and P. Bremner. Social robots for engagement in rehabilitative therapies: Design implications from a study with therapists. In Proceedings of the 2018 acm/ieee international conference on human-robot interaction, pages $289-297,2018$.

[36] B. Woodworth, F. Ferrari, T. E. Zosa, and L. D. Riek. Preference learning in assistive robotics: Observational repeated inverse reinforcement learning. In Machine Learning for Healthcare Conference, pages 420-439, 2018.

[37] J. Złotowski, H. Sumioka, S. Nishio, D. F. Glas, C. Bartneck, and H. Ishiguro. Appearance of a robot affects the impact of its behaviour on perceived trustworthiness and empathy. Paladyn, fournal of Behavioral Robotics, 1(open-issue), 2016. 